\title{
TWO ALGEBRAIC IDENTITIES AND THE UNBOUNDEDNESS OF A RESTRICTED PARTITION FUNCTION
}

\author{
CULBRETH SUDLER, JR.
}

1. Introduction. We consider in this note the polynomial

$$
\prod_{k=1}^{n}\left(1-x^{k}\right)=\sum_{h} a_{n h} x^{h}
$$

For the corresponding infinite product

$$
\prod_{k=1}^{\infty}\left(1-x^{k}\right)=\sum_{h} C_{h} x^{h}
$$

the coefficients $C_{h}$ are given by the "pentagonal number" theorem of Euler [2, p. 284]. $C_{h}$ also has a combinatorial definition as follows. Let $P=\left(b_{1}, \cdots, b_{v}\right)$ be a partition of $h$ into unequal summands $b_{i}, 1 \leqq i \leqq \nu$. Then $C_{h}=\sum_{P}(-1)^{\nu}$, the summation being taken over all partitions $P$. Moreover, the Euler identity can be proved directly from this definition [2, Chapter 19]. $a_{n h}$ has an analogous definition in which the largest summand in any partition $P$ is $\leqq n$. Hence $a_{n h}=C_{h}$ for $h \leqq n$.

Since the $C_{h}$ are bounded, it is of interest to determine whether the $a_{n h}$ are bounded for all $n$, a problem raised by Nicol $[3$, p. 38]. In [4] we have given a negative answer by showing that $\log \max _{h}\left|a_{n h}\right| \sim K n$ for a constant $K>0$. Here we obtain two identities which yield an unbounded sequence of values of $a_{n h .}{ }^{1}$ We will show that

$$
\prod_{k=1}^{n}\left(1-x^{k}\right)=\sum_{r=0}^{\infty} x^{r(n+1)} \prod_{m=r+1}^{\infty}\left(1-x^{m}\right),
$$

and for $r \geqq 1$,

$$
\begin{aligned}
\prod_{m=r+1}^{\infty}\left(1-x^{m}\right)= & \sum_{t=0}^{\infty}(-1)^{t} x^{t(3 t+2 r-1) / 2} \cdot\left(1-x^{2 t+r}\right) \\
& \cdot \prod_{s=1}^{r-1}\left(1-x^{t+s}\right) \prod_{s=1}^{r}\left(1-x^{s}\right)^{-1}
\end{aligned}
$$

In order to exhibit unbounded values of $a_{n h}$, (1.4) will be utilized only for $r=1$ and 2. It is convenient, however, to state the general result which is a special case of a known identity. For convergence,

Received by the editors October 18, 1962.

1 Professor T. S. Motzkin of the University of California, Los Angeles, obtained this result by a different method in 1955 . His work is still unpublished. 
we assume in the sequel that $|x|<1$ and $|a|<1$. The index of a sum or product will always be in the exponents or subscripts of its terms, and when there is no risk of ambiguity, it will be omitted. When unspecified, the range of a sum is 0 to $\infty$, and the empty product is to be interpreted as unity.

2. Proof of (1.3) and (1.4). We have

$$
\prod_{1}^{n}\left(1-x^{k}\right)=\prod_{1}^{\infty}\left(1-x^{k}\right) \prod_{n+1}^{\infty}\left(1-x^{l}\right)^{-1} .
$$

In Theorem 349 of Hardy and Wright [2] we let $j \rightarrow \infty$ and deduce that

$$
\prod_{1}^{\infty}\left(1-a x^{k}\right)^{-1}=\sum_{i}(a x)^{i} \prod_{1}^{i}\left(1-x^{k}\right)^{-1}
$$

Putting $a=x^{n}$ gives now

$$
\begin{aligned}
\prod_{n+1}^{\infty}\left(1-x^{l}\right)^{-1} & \\
& =1+x^{n+1} /(1-x)+x^{2 n+2} /(1-x)\left(1-x^{2}\right)+\cdots .
\end{aligned}
$$

On substituting (2.3), the right member of (2.1) becomes

$$
\begin{aligned}
\left(\prod_{1}^{\infty}\left(1-x^{k}\right)\right)\left(\sum_{r} x^{r(n+1)} \prod_{1}^{r}\left(1-x^{s}\right)^{-1}\right) \\
=\sum_{r} x^{r(n+1)} \prod_{k=r+1}^{\infty}\left(1-x^{k}\right) .
\end{aligned}
$$

(2.4) and (2.1) now give (1.3).

To obtain (1.4) we put $b=x^{r}$ in the following identity of Cayley and Sylvester $[1$, p. 140], where $|b|<1$.

$$
\begin{aligned}
\prod_{1}^{\infty}(1 & \left.-b x^{k}\right) \\
& =1+\sum_{1}^{\infty}(-b)^{t} x^{t(8 t-1) / 2}\left(1-b x^{2 t}\right)\left(\prod_{1}^{t-1}\left(1-b x^{b}\right)\right) / \Pi_{t},
\end{aligned}
$$

where $\Pi_{t}=\prod_{1}^{t}\left(1-x^{s}\right)$. In order to verify our assertion, we note that by cross-multiplication,

$$
\prod_{s=1}^{r-1}\left(1-x^{t+s}\right) / \Pi_{r}=\prod_{s=1}^{t-1}\left(1-x^{r+s}\right) / \Pi_{t},
$$

where the quotient on the left is that in (1.4). (1.4) was originally 
conjectured by examining the special cases $r=1$ and 2, the form of which was guessed on multiplying the right member of (1.2) by $(1-x)$ and $(1-x)\left(1-x^{2}\right)$, respectively, and inspecting the resulting sequence of coefficients.

Since (2.5) seems to be unfamiliar, a short proof is given below. Dividing through by $\prod_{1}^{\infty}\left(1-b x^{k}\right)$ gives $1=\sum_{0}^{\infty} b^{u} g_{u}(x)$, say, where $g_{u}(x)$ is a function of $x$ only, and $g_{0}(x)=1$. Since

$$
\prod_{t}^{\infty}\left(1-b x^{k}\right)^{-1}=\sum_{i} b^{i} x^{t i} / \Pi_{i}, \quad t \geqq 0
$$

by (2.2) with $a$ replaced by $b x^{t-1}$, we find after a little reduction that for $u \geqq 1$,

$$
\begin{aligned}
g_{u}(x)= & \sum_{0}^{u}(-1)^{t} x^{t(u-1)+t(t+1) / 2} /\left(\Pi_{t} \cdot \Pi_{u-t}\right) \\
& -\sum_{0}^{u-1}(-1)^{t} x^{t u+(t+1) / 2} /\left(\Pi_{t} \cdot \Pi_{u-t-1}\right) \\
= & \prod_{1}^{u}\left(1-x^{l+u-1}\right) / \Pi_{u}-\prod_{1}^{u-1}\left(1-x^{l+u}\right) / \Pi_{u-1}
\end{aligned}
$$

by [2, Theorem 348], and the last expression is 0 . This completes the proof of (2.5).

3. Proof that $a_{n h}$ is unbounded. Denote the left member of (1.4) by $P_{r}(x)$ and put

$$
P_{r}(x)=\sum_{m} C_{r m} x^{m}
$$

Then we have by (1.3),

$$
a_{n h}=\sum_{0}^{[h /(n+1)]} C_{r, h-r(n+1)} .
$$

In order to deduce that $a_{n h}$ is unbounded, it suffices to find a minimum value of $r$ such that the numbers $C_{r m}$ of (3.1) are unbounded. Now $P_{0}(x)$ has bounded coefficients by the Euler identity. We will show that $C_{1, m}$ is bounded and $C_{2, m}$ is unbounded. By (1.4)

$$
P_{1}(x)=\sum(-1)^{t} x^{t(3 t+1) / 2}\left(1-x^{2 t+1}\right) /(1-x)
$$

and

(3.4) $\quad P_{2}(x)=\sum(-1)^{t} x^{t(3 t+3) / 2}\left(1-x^{t+1}\right)\left(1-x^{2 t+2}\right) /(1-x)\left(1-x^{2}\right)$.

We prove first that the summands of the right member of (3.4) 
are polynomials with unbounded coefficients. It is sufficient to prove this for

$$
f_{t}(x)=\left(1-x^{t+1}\right)\left(1-x^{2 t+2}\right) /(1-x)\left(1-x^{2}\right)
$$

which is evidently a polynomial. Now $f_{t}(1)=(t+1)^{2}$ and $f_{t}(x)$ is of degree $3 t$, so that its coefficients are unbounded by Dirichlet's box principle. Next, it is easily seen that for $r=1$ and 2, $C_{r m}$ is obtained from the expansion of a single summand of the right member of (3.3) or (3.4). Hence $C_{1, m}$ is bounded and $C_{2, m}$ is unbounded. We now utilize (3.2) to obtain our final result.

If $h \leqq 2 n+1, r=0,1$ only in (3.2) and $a_{n h}$ is bounded for $h$ in this range. Suppose now that $2 n+2 \leqq h \leqq 3 n+2$. Then

$$
a_{n h}=C_{2, h-(2 n+2)}+O(1) .
$$

Put $R=h-(2 n+2)$. Then $a_{n h}$ is unbounded as

$$
n \rightarrow \infty, \quad R \rightarrow \infty, \quad R<n+1 .
$$

Furthermore, (1.4) and (1.3) provide a relatively simple explicit formula for $a_{n h}$ when $h \leqq 3 n+2$.

For all $r$, the summands of (1.4) are polynomials, a proof of which we indicate for completeness. Each summand is a quotient $f(x) / g(x)$ of two polynomials $f(x)$ and $g(x)$, the zeros $\rho$ of which are roots of unity. We count the multiplicity $M_{0}(\rho)$ of any zero of $g(x)$ and show easily that $M_{0}(\rho) \leqq M_{f}(\rho)$, the corresponding multiplicity for $f(x)$. However, for $r \geqq 3$ the numbers $C_{r m}$ of (3.1) are not obtained from a single summand of (1.4).

The method of proof in this paper was obtained after I read a reference to Motzkin's result. I wish to express my gratitude to him for encouraging the publication of my paper and to the referee for pointing out several obscurities. The proof of (1.4) contained herein was discovered after this paper had been accepted for publication and replaces an earlier and much less efficient verification.

Added in proof. Dr. John B. Kelly of Arizona State University has pointed out to me that we can dispense with (3.4) in proving that $C_{2, m}$, and hence $a_{n h}$, is unbounded. His argument runs as follows.

On dividing through by $1-x$ in (3.3), it is clear that the sequence $C_{1, m}$ consists of blocks of consecutive terms separated by zeros such that the $t$ th such block contains exactly $2 t-1$ terms all equal to $(-1)^{t-1}$. By the definition of $P_{r}(x)$, we have $P_{2}(x)=P_{1}(x) /\left(1-x^{2}\right)$, and the coefficient of $x^{m}$ in the expansion of $P_{2}(x)(1+x)$ is 


$$
S=\sum_{0}^{m} C_{1, \mu}=C_{2, m}+C_{2, m-1}
$$

Choose $m=m(t)$ so that $C_{1, m}$ is the last term of the $t$ th block. Then $|S|=t$ and $\operatorname{Max}\left(C_{2, m}, C_{2, m-1}\right)$ is unbounded, which gives the result.

\section{REFERENCES}

1. L. E. Dickson, History of the theory of numbers, Vol. II, Chelsea, New York, 1952.

2. G. H. Hardy and E. M. Wright, An introduction to the theory of numbers, 4th ed., Oxford Univ. Press, Oxford, 1960.

3. C. A. Nicol and H. S. Vandiver, On generating functions for restricted partitions of rational integers, Proc. Nat. Acad. Sci. (U.S.A.) 41 (1955), 37-42.

4. C. Sudler, Jr., An estimate for a restricted partition function, Quart. J. Math. Oxford Ser. (2) (to appear).

Los Angeles, California 